Theories \& Applications, the International Edition

Printed Version : (ISSN 2090-5262)

Online Version : (ISSN 2090-5270)

July 2014, Volume 4, No. 2 Pages (103 - 116)

\title{
Effect of Different Intensity Step Exercises on some Physical Abilities and Functional Responses and Performance Level for Divers.
}

\section{Dr. Karim Ahmed Ibrahim Shehata}

lecturer, aqua sports training department, Faculty of physical education for men, Alexandria university, Egypt.

\begin{abstract}
The research aims to design training program using varying intensity step exercises accompanied with music and its impact on certain physical abilities, some functional responses, and improving performance level .

Study was conducted on intentionally selected sample consisted of (25) students from third-grade students at Faculty of Physical Education for Men, who practice amateur diving as juniors, that is because diving is one of academic syllabuses for this grade divided to an experimental group of (10) students who undergone the proposed different intensity step secrecies program accompanied with music (60-75\% of pulse reserves time) and a control group of (10) students who undergone followed diving teaching program for third students and did not participate in proposed training program. Researcher taught Scuba unit put on/off skill underwater for both groups (control-experimental) - also taught different intensity step exercises accompanied with music to experimental group only through an educational program for a period of two weeks conducted (4 sessions/week), after this main study, later main study conducted for period of (10 weeks) by (3 sessions/week) and session time (60 minutes) at swimming pool of faculty of physical education for men, Alexandria university, results reveal superiority of experimental group over control group in certain physical abilities, some functional responses, and performance level, so the researcher recommends using different intensity step exercises due to its positive effect on developing some physical abilities , functional responses, and improving performance level for various physical activities.
\end{abstract}

\section{Introduction:}

$\mathrm{D}$ ving sport encourage studies for best training ways and methods, which can be used to improve some physical and physiological abilities necessary for practicing this type of activity. Diving is classified within physical activities which affected by several variables at same time. It depends on multiple factors for selecting most appropriate structured training methods, which is one of the most important ingredient that cause improvement in some physical abilities and functional efficiency of main body organs such as the circular and respiratory systems and working muscles in the body, Diver proficiency is determined his physical physiological abilities

As physical and physiological facts is the basis for directing training process with what commensurate with each sporting activity to access a level of achievement. Diving have got sophisticated and significant interest in many institutions and organizations due to its important economic aspect, as it is an important element of national income, in the same time recent years, scientific have had remarkable progress in training field generally and in diving particularly.

MagdiyAbu Zaid (2007, p186-187) argues that diving is a special nature sport, as it requires certain characteristics to get diver do his work safely under water. Diver should be characterized with high and physical of physiological abilities to enable him to perform his tasks skillfully and mastery.
Recently, diving training methods to improve some physical abilities and functional responses as a basis to raise performance level, Levy, Furest (2002, p137) mentioned that there are many research confirm the positive impact of step exercises on improving and fitness of cardio respiratory system, and increase cardiac output and blood circulation with effort saving.

Brick (2001, p2) argues that step exercises have effective effect on improving fitness level, as well as helping body's systems to work more efficiently, especially the lower limb muscles. (9:2)

Afaf Darwish and Nourhanne Hassan (1997, p13) adds that step exercises using step box are considered latest types of aerobic exercise which depends going ups and downs from the box, so it increase player's enthusiasm and acquire him performance challenge through his moves on the box with different changes in his body parts t. (5: 13)

Corbin and Lindsey (2000, p73) and Champion, Hurst (2000, p96) agreed that carefully planned step exercises would be influential in development and construction of cardio respiratory fitness, and hence possibility of continuing performance for long periods before reaching fatigue stage.

Mahmoud Marei (2001, p120), Iman Abdullah (2000, p84) Abeer Ramadan (2000, p82) and Azmi Faisal (2002, p163) studies confirmed the positive effects of step exercise on physical aspects like neuromuscular endurance, agility, motor physical in addition the muscular endurance of legs, 
abdomen, and arms muscles . (7: 120), (1: 84), (3: 82), (4: 163)

Scientific researches vision for the positive effect of step exercises on improving some physical abilities and functional responses called the researcher to conduct this study to identify the impact of different intensity step exercises accompanied with music on some physical abilities, functional responses and skills performance level, and then directing training program to achieve better level for diving juniors.

\section{Research objectives:}

The research aims to design training program using varying intensity step exercises accompanied with music and its impact on:
A- Certain physical abilities.
B- Some functional responses.
C- Improving performance level.

\section{Research hypotheses:}

1- There are statistically significant differences between pre and post measurements in some physical abilities level for experimental and control group in favor of experimental group.

2- There are statistically significant differences between pre and post measurements in some functional responses level for experimental and control group in favor of experimental group.
3- There are statistically significant differences between pre and post measurements in improving performance time for experimental and control group in favor of experimental group.

\section{Research domains:}

\section{Spatial domain:}

Swimming pool, exercises lounge and gymnastics of Faculty of Physical Education for Men, and Chest Department (respiratory functions), Faculty of Medicine, Alexandria University.

\section{Time domain:}

The period from Three months,

\section{Research sample:}

Study was conducted on intentionally selected sample consisted of (25) students from third-grade students at Faculty of Physical Education for Men, who practice amateur diving as juniors, that is because diving is one of academic syllabuses for this grade distributed as follows:

- An experimental group of (10) students who undergone the proposed different intensity step secrecies program accompanied with music $(60-75 \%$ of pulse reserves time).

- A control group of (10) students who undergone followed diving teaching program for third students and did not participate in proposed training program Both groups undergone pre- and post-measurements.

- Pilot study group of (5) students.

Table (1)

Statistical description of experimental and control groups and total research sample in basic measurements prior experiment.

\begin{tabular}{|c|c|c|c|c|c|c|}
\hline \multirow{2}{*}{ measurements } & \multirow{2}{*}{ Groups } & \multirow{2}{*}{$\mathbf{N}$} & \multicolumn{4}{|c|}{ Statistical description significance } \\
\hline & & & Mean & Median & SD & Skweness coefficient \\
\hline \multirow{3}{*}{ Age to nearest month } & Experimental & 10 & 19.65 & 20 & 1.34 & -0.62 \\
\hline & Control & 10 & 19.93 & 20 & 0.24 & -0.16 \\
\hline & $\begin{array}{c}\text { Total research } \\
\text { sample }\end{array}$ & 20 & 19.79 & 20 & 0.95 & -0.59 \\
\hline \multirow{3}{*}{ Height $\mathrm{cm}$} & Experimental & 10 & 174.2 & 175 & 3.49 & -1.39 \\
\hline & Control & 10 & 175.1 & 173 & 5.22 & 0.56 \\
\hline & $\begin{array}{c}\text { Total research } \\
\text { sample } \\
\end{array}$ & 20 & 174.65 & 175 & 4.34 & 0.24 \\
\hline \multirow{3}{*}{ Weight kg } & Experimental & 10 & 77.5 & 78 & 4.22 & -1.19 \\
\hline & Control & 10 & 78.9 & 77 & 7.95 & 0.3 \\
\hline & $\begin{array}{c}\text { Total research } \\
\text { sample }\end{array}$ & 20 & 78.2 & 78 & 6.24 & 0.32 \\
\hline
\end{tabular}

Table (1) results reveal that skewness factor for all variables ranged between ( -1.39 to 0.56$)$ i.e. between \pm 3 and near to zero, indicating that all obtained measurements are close to normal distribution. which confirms homogeneity of experimental and control groups, and total research sample in basic variables prior to the experiment. 
Table (2)

Differences between experimental and control groups in basic measurements prior experiment (homogeneity)

\begin{tabular}{|c|c|c|c|c|c|c|}
\hline \multirow{2}{*}{ measurements } & \multicolumn{2}{|c|}{ Experimental group $(n=10)$} & \multicolumn{2}{|c|}{ Control group $(n=10)$} & \multirow{2}{*}{$\begin{array}{l}\text { Differences } \\
\text { between } \\
\text { averages }\end{array}$} & \multirow{2}{*}{ T value } \\
\hline & Mean & SD & Mean & SD & & \\
\hline Age to nearest month & 19.65 & 1.34 & 19.93 & 0.24 & 0.28 & 0.64 \\
\hline Height $\mathrm{cm}$ & 174.2 & 3.49 & 175.1 & 5.22 & 0.9 & 0.45 \\
\hline Weight kg & 77.5 & 4.22 & 78.9 & 7.95 & 1.4 & 0.49 \\
\hline
\end{tabular}

* T Significant at the 0.05 level $=\mathbf{2 . 1 0}$

Table (2) results reveal that $\mathrm{T}$ value for differences between experimental and control groups in the basic measurements ranged from ( 0.45 to 0.64$)$ and these values were not significant at the 0.05 level, which indicates that the two research groups are homogeneous in basic measurements.

Research Tools:

\section{I - Tools and equipments:}

Restameter for measuring height $(\mathrm{cm})$, medical calibrated scale for measuring weight $(\mathrm{kg}$,) Polar watch to measure the pulse during physical exertion in training sessions, simple reaction device, Chest Graph HI-701 device for measuring pulse at rest (electronic), Geneometer device (for measuring motionrange) Mternom (to adjust the rhythm), HP laptop equipped with recorded musical rhythms begin from 60 beat/minute to 110 beat/minute Sony speakers with high frequency (1000 RMS), diving goggles (Mask), diving fins, diving suits (dry), air cylinders (oxygen), air regulator, lead belts, tape measure $(\mathrm{cm})$ buoy (phosphoric), lead weight, $20 \mathrm{~cm}$ hieight step box .

II- Physical, functional and performance time tests and measurements:

Physical, functional and performance time tests and measurements carried out for both groups (experimental - control) prior to experiment, and post the experiment ,

\section{1- Physical measurements and tests:}

A- special endurance test:

- $400 \mathrm{~m}$ fins swimming as it contribute to energy production systems ( $\mathrm{min}$ ).

\section{B- Muscle strength endurance:}

- Knee bent from stand test (40 sec) to measure legs' muscular endurance

- Setting from squatting lying test (40 sec) to measure abdominal and thigh flexor muscles muscular endurance.

- Trunk lifting from prostration (40 sec to measure muscular endurance.

- Arms bend from slanted prostration amended test $(40 \mathrm{sec})$ to measure arms and shoulders muscular endurance.

\section{C- Motion Range measurements:}

- Measuring hip motion range in extending and adduction (Geneometer).

- Measuring foot motion range in extending and adduction (Geneometer).

D- Motor speed tests:

- Pendulum running test $4 \times 9 \mathrm{~m}(\mathrm{sec})$ to measure legs motor speed.

- Measuring simple reaction speed ( reaction device).

E- Power

- Vertical jump test to measure legs muscle ability.

F- Spatial direction ability test (agility under water) (sec).

2- Functional responses are as follows:

A - Lung Function Tests include:

- Vital capacity (VC).

- Forced vital capacity (FVC).

- Forced expiratory volume in first second (FEV1).

- Forced expiratory volume in the first second\% FEV1\%).

- Peak Expiratory Flow Rate (PEFR).

- Forced aspiratory vital capacity (FIVC).

- Maximum voluntary ventilation (MVV).

B - Respiratory fitness tests include:

- Resting pulse (pulse/sec).

- Physical work efficiency at pulse of 170 PWC 170 (W) (Kariman test).

- Relative Physical work efficiency at pulse of 170 PWC 170 (Watt/Kg)).

- Maximum oxygen consumption (VO2 MAX) (Quinn test).

3- Scuba unit put on/off skill underwater time measurement

Pilot study: 
The researcher conducted a pilot study on sample of (5) students in order to determine the appropriate step box height and step exercises various intensities accompanying music, which can be formed from training loads for the proposed program, Faisal. A, et al (2009, p143) indicated that pulse rates are stabled after 2- 3 minutes from the beginning of medium intensity physical effort, so time of pulse measurements for arms and legs movements using step box has been determined as ( 3 minutes) after starting, height $(20 \mathrm{~cm})$, where the researcher used the Polar watch to measure and record pulse during performance.

\section{Main study:}

The researcher taught Scuba unit put on/off skill underwater for both groups (control-experimental) - also taught different intensity step exercises accompanied with music to experimental group only through an educational program for a period of two weeks, with (4 sessions/week) each session time is 45 minutes, later the main study conducted . for ( 10 weeks) by ( 3 sessions/week) and session time (60 minutes), where experimental group perform various intensity (50$70 \%$ ) step exercise, and control group perform regular diving syllabus for $3^{\text {rd }}$ grade all done inside swimming pool lounge at Faculty of Physical Education for Men, Alexandria University, where each group (experimental control) performed its training dose as per training foundations and training physiological laws..

\section{Proposed training program general principles:}

Based on the scientific consensus [Sharkey (2001, p89), Foss \& Keteyian $(2002,176)$ and Bastawissi Ahmad Bastawissi (1999, p99)] researcher has identified proposed training program general principles for going up/down the step box accompanied with music to develop :physical and physiological abilities level, and Scuba unit put on/off skill time measurement for experimental group as follows:

- Principle of load and in-between rest as training basis.

- Wave principle in training load dynamic in terms of increase and decrease load intensity for both training session-level, and training program as a whole.

- Individual training principle so training load intensity is determined by player's pulse reserve according to training's functional direction, based on Carfoden formula for aimed training pulse calculating.

\section{Proposed training program variables:}

- Load volume: determined by various intensity step exercises proposed program's duration, weekly training session, and training session time, step exercises time in session and in all sessions.

- Load intensity: determined pulse reserve depending on training functional direction for experimental group, where its rate ranged between $50-70 \%$ of pulse reserves. $\circ$ Where reserves pulse $=$ maximum pulse - rest pulse

○ Maximum pulse $=220$-age

- Using Carfoden formula aimed training pulse could be calculated as follow:

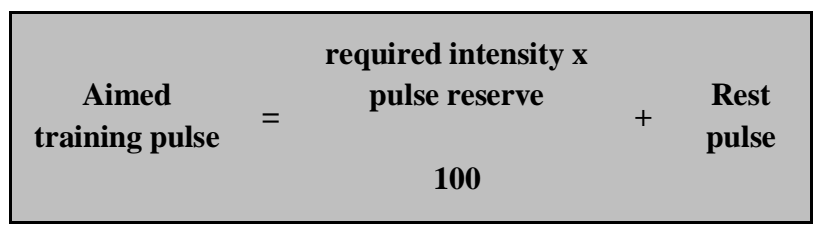

- In-between-rest: determined as 1:3 of training duration so student do going up/down the step box for (3 minutes) to reach required pulse rates, followed by lower intensity for (1 minute), where pulse rate is reduced to $115-110$ beats (positive rest principle with partial rest times) to achieve:

- Partial positive rest from previous intensity.

- Maintain intensity continuation and use various intensity wave load principle.

\section{Training session content:}

Training session of (60) minutes included the following:

\section{Warm-up (10 minutes):}

This part included various running exercise so pulse rate reach 110-115 beats/minute and stretching exercises of various muscle groups.

\section{Physical preparation (31 minutes):}

This party included various intensity step exercises accompanied with music, where intensity rate through the program ranged between $50-70 \%$ of pulse reserves, and training load formed using various intensity waves through training session ( (variable load).

\section{Skill preparation (15 mminuts):}

Student performs Scuba unit put on/off skill underwater as follow:
A- Scuba unit put off skill underwater
B- Scuba unit put on skill underwater

\section{Calling Down: (4 minutes):}

This part included swinging and stretching exercises

Educational program for Scuba unit put on/off skill underwater and different intensity step exercises accompanied with music:

Both research groups (experimental control) taught Scuba unit put on/off skill underwater, while different intensity step exercises accompanied with music taught to experimental group only, through an educational program for ( 2 weeks) with 4 sessions/week, session time is of 45 minutes as shown in tale (3): 
Table (3)

Time schedule for Scuba unit put on/off skill and different intensity step exercises accompanied with music

\begin{tabular}{|c|c|c|c|}
\hline Week & Session aim & Research groups & Session time \\
\hline \multirow{4}{*}{$1^{\text {st }}$} & $\begin{array}{l}\text { Scuba unit put on skill } \\
\text { (out of water) }\end{array}$ & Experimental-control & 45 minutes \\
\hline & Teaching some different intensity step exercises & Experimental & 45 minutes \\
\hline & $\begin{array}{l}\text { Scuba unit put off skill } \\
\text { (out of water) }\end{array}$ & Experimental-control & 45 minutes \\
\hline & Teaching some different intensity step exercises & Experimental & 45 minutes \\
\hline \multirow{4}{*}{$2^{\text {nd }}$} & $\begin{array}{l}\text { Scuba unit put on skill } \\
\text { (out of water) }\end{array}$ & Experimental-control & 45 minutes \\
\hline & $\begin{array}{c}\text { Associating different intensity step exercises with } \\
\text { accompanied music }\end{array}$ & Experimental & 45 minutes \\
\hline & Scuba unit put on/off skill underwater & Experimental-control & 45 minutes \\
\hline & $\begin{array}{l}\text { performing different intensity step exercises with } \\
\text { accompanied music }\end{array}$ & Experimental & 45 minutes \\
\hline
\end{tabular}

\section{Post-measurements:}

Post-measurements was performed for both research groups (experimental - control), after completion of proposed program application with same conditions of prmeasurements.

\section{Statistical work:}

In light of research variables, the researcher used the following statistical factors/tests:

$$
\text { - Mean. }
$$

- Standard deviation .

- Skewness coefficient

- Independent $\mathrm{T}$ test for identifying difference significance between two homogeneous groups

- Paired T test for identifying difference significance between pre and post-measurements for same group

All statistical work done using Statistical Package for Social Sciences (SPSS)

Results

Table (4)

statistical characterization for experimental, control and overall research sample in physical tests before the experiment

\begin{tabular}{|c|c|c|c|c|c|c|c|}
\hline \multirow[t]{2}{*}{ Statistics } & \multirow[b]{2}{*}{ Tests } & \multirow[b]{2}{*}{ Groups } & \multirow[b]{2}{*}{$\mathbf{N}$} & \multicolumn{4}{|c|}{ Statistics significance } \\
\hline & & & & Mean & Median & $\begin{array}{l}\text { Slandered } \\
\text { Deviation }\end{array}$ & $\begin{array}{l}\text { Skweness } \\
\text { coefficient }\end{array}$ \\
\hline \multirow{12}{*}{$\begin{array}{c}\text { Muscle strength } \\
\text { endurance }\end{array}$} & \multirow{3}{*}{$\begin{array}{c}400 \mathrm{~m} \\
\text { swimming } \\
\text { time }\end{array}$} & Experimental & 10 & 8.21 & 8.32 & 0.56 & -0.84 \\
\hline & & Control & 10 & 8.34 & 8.4 & 0.56 & $\mathbf{0}$ \\
\hline & & Overall & 20 & 8.27 & 8.32 & 0.55 & -0.37 \\
\hline & \multirow{3}{*}{ Knee bent } & Experimental & 10 & 48 & 50 & 7.26 & -1.14 \\
\hline & & Control & 10 & 49.9 & 51 & 7.02 & 0.2 \\
\hline & & Overall & 20 & 48.95 & 51 & 7.01 & -0.47 \\
\hline & \multirow{3}{*}{$\begin{array}{c}\text { Setting } \\
\text { from lying }\end{array}$} & Experimental & 10 & 36.8 & 37.5 & 5.53 & -0.1 \\
\hline & & Control & 10 & 35.4 & 34.5 & 4.74 & -0.06 \\
\hline & & Overall & 20 & 36.1 & 36 & 5.07 & -0.01 \\
\hline & \multirow{3}{*}{ Trunk lifting } & Experimental & 10 & 33.6 & 34.5 & 7.18 & 0.03 \\
\hline & & Control & 10 & 33.9 & 33 & 7.99 & -0.19 \\
\hline & & Overall & 20 & 33.75 & 34 & 7.4 & -0.08 \\
\hline \multirow{4}{*}{ Motion Range } & \multirow{3}{*}{ Arms bend } & Experimental & 10 & 21.9 & 21.5 & 3.11 & 0.5 \\
\hline & & Control & 10 & 20.4 & 20 & 3.34 & 0.06 \\
\hline & & Overall & 20 & 21.15 & 21 & 3.23 & 0.16 \\
\hline & Foot & Experimental & 10 & 29.6 & 29 & 6.75 & -0.01 \\
\hline
\end{tabular}




\begin{tabular}{|c|c|c|c|c|c|c|c|}
\hline \multirow[t]{2}{*}{ Statistics } & \multirow[b]{2}{*}{ Tests } & \multirow[b]{2}{*}{ Groups } & \multirow[b]{2}{*}{$\mathbf{N}$} & \multicolumn{4}{|c|}{ Statistics significance } \\
\hline & & & & Mean & Median & $\begin{array}{l}\text { Slandered } \\
\text { Deviation }\end{array}$ & $\begin{array}{l}\text { Skweness } \\
\text { coefficient }\end{array}$ \\
\hline & \multirow[t]{2}{*}{ Adduction } & Control & 10 & 27.2 & 25 & 5.81 & 0.39 \\
\hline & & Overall & 20 & 28.4 & 27.5 & 6.25 & 0.22 \\
\hline & \multirow{3}{*}{$\begin{array}{c}\text { Foot } \\
\text { extending }\end{array}$} & Experimental & 10 & 26.6 & 25 & 7.6 & 0.61 \\
\hline & & Control & 10 & 25.5 & 27.5 & 4.97 & -0.24 \\
\hline & & Overall & 20 & 26.05 & 27.5 & 6.28 & 0.52 \\
\hline & \multirow{3}{*}{$\begin{array}{c}\text { Hip } \\
\text { adduction }\end{array}$} & Experimental & 10 & 42.7 & 40.5 & 6.7 & 2.2 \\
\hline & & Control & 10 & 40 & 41.5 & 8.55 & -0.47 \\
\hline & & Overall & 20 & 41.35 & 40.5 & 7.6 & 0.21 \\
\hline & \multirow{3}{*}{$\begin{array}{c}\text { Hip } \\
\text { Extending }\end{array}$} & Experimental & 10 & 86.2 & 83.5 & 8.39 & 0.36 \\
\hline & & Control & 10 & 82.8 & 78.5 & 8.05 & 0.78 \\
\hline & & Overall & 20 & 84.5 & 83 & 8.19 & 0.5 \\
\hline \multirow{6}{*}{ Speed } & \multirow{3}{*}{$\begin{array}{c}\text { Pendulum } \\
\text { Running }\end{array}$} & Experimental & 10 & 10.75 & 10.65 & 0.53 & 0.1 \\
\hline & & Control & 10 & 10.53 & 10.57 & 0.47 & -0.42 \\
\hline & & Overall & 20 & 10.64 & 10.58 & 0.5 & -0.02 \\
\hline & \multirow{3}{*}{$\begin{array}{c}\text { reaction } \\
\text { speed }\end{array}$} & Experimental & 10 & 0.23 & 0.2355 & 0.02 & 0.02 \\
\hline & & Control & 10 & 0.23 & 0.233 & 0.01 & 0.03 \\
\hline & & Overall & 20 & 0.23 & 0.2335 & 0.02 & 0.14 \\
\hline \multirow{3}{*}{ Power } & \multirow{3}{*}{$\begin{array}{l}\text { Vertical } \\
\text { jump }\end{array}$} & Experimental & 10 & 32.9 & 33.5 & 3.38 & -0.28 \\
\hline & & Control & 10 & 33.2 & 33.5 & 3.39 & -0.26 \\
\hline & & Overall & 20 & 33.05 & 33.5 & 3.3 & -0.24 \\
\hline \multirow{3}{*}{ Ability } & \multirow{3}{*}{$\begin{array}{c}\text { Agility } \\
\text { under water }\end{array}$} & Experimental & 10 & 11.8 & 11.645 & 1.29 & 0.03 \\
\hline & & Control & 10 & 12.14 & 12.485 & 1.63 & -0.24 \\
\hline & & Overall & 20 & 11.97 & 11.955 & 1.44 & -0.05 \\
\hline
\end{tabular}

Table (4) results show that skewness coefficient range from ( -1.14 to 2.20$)$, indicating that measurements obtained are close to normal distribution where skewness coefficient values should be between \pm 3 and very close to zero This confirms homogeneity of experimental, control, and overall research sample in physical tests before the experiment .

Table (5)

statistical characterization for experimental, control and overall research sample in physiological measurements before the experiment

\begin{tabular}{|c|c|c|c|c|c|c|c|}
\hline \multirow[t]{2}{*}{ Statistics } & \multirow[b]{2}{*}{ Tests } & \multirow[b]{2}{*}{ Groups } & \multirow[b]{2}{*}{$\mathbf{N}$} & \multicolumn{4}{|c|}{ Statistics significance } \\
\hline & & & & Mean & Median & $\begin{array}{l}\text { Slandered } \\
\text { Deviation }\end{array}$ & $\begin{array}{l}\text { Skweness } \\
\text { coefficient }\end{array}$ \\
\hline \multirow{21}{*}{$\begin{array}{c}\text { Lung } \\
\text { Function } \\
\text { Tests }\end{array}$} & \multirow{3}{*}{ V C } & Experimental & 10 & 7.09 & 7.04 & 0.65 & 0.65 \\
\hline & & Control & 10 & 6.97 & 6.66 & 1.2 & 1.28 \\
\hline & & Overall & 20 & 7.03 & 6.94 & 0.94 & 1.08 \\
\hline & \multirow{3}{*}{ F V C } & Experimental & 10 & 7.03 & 7.04 & 0.61 & 0.98 \\
\hline & & Control & 10 & 6.75 & 6.57 & 1.12 & 2.02 \\
\hline & & Overall & 20 & 6.89 & 6.94 & 0.89 & 1.55 \\
\hline & \multirow{3}{*}{ F E V 1} & Experimental & 10 & 5.93 & 5.87 & 0.52 & 1.51 \\
\hline & & Control & 10 & 5.75 & 5.775 & 1 & 0.21 \\
\hline & & Overall & 20 & 5.84 & 5.87 & 0.78 & 0.17 \\
\hline & \multirow{3}{*}{ FEV $1 \%$} & Experimental & 10 & 84.48 & 84.705 & 5.94 & 0.63 \\
\hline & & Control & 10 & 85.51 & 90.67 & 9.64 & -0.55 \\
\hline & & Overall & 20 & 84.99 & 85.555 & 7.81 & -0.22 \\
\hline & \multirow{3}{*}{ P E F } & Experimental & 10 & 12.11 & 11.77 & 1.27 & -0.07 \\
\hline & & Control & 10 & 12.27 & 12.25 & 1.17 & -0.25 \\
\hline & & Overall & 20 & 12.19 & 12.11 & 1.19 & -0.15 \\
\hline & \multirow{3}{*}{ F I V C } & Experimental & 10 & 6.83 & 7.01 & 0.71 & -0.94 \\
\hline & & Control & 10 & 6.5 & 6.55 & 0.61 & 0.18 \\
\hline & & Overall & 20 & 6.67 & 6.755 & 0.67 & -0.3 \\
\hline & \multirow{3}{*}{ M V V } & Experimental & 10 & 182 & 178.4 & 32.91 & 0.08 \\
\hline & & Control & 10 & 169.53 & 173.2 & 32.46 & 0.74 \\
\hline & & Overall & 20 & 175.77 & 175.5 & 32.45 & 0.35 \\
\hline
\end{tabular}




\begin{tabular}{|c|c|c|c|c|c|c|c|}
\hline \multirow[t]{2}{*}{ Statistics } & \multirow[b]{2}{*}{ Tests } & \multirow[b]{2}{*}{ Groups } & \multirow[b]{2}{*}{$\mathbf{N}$} & \multicolumn{4}{|c|}{ Statistics significance } \\
\hline & & & & Mean & Median & $\begin{array}{l}\text { Slandered } \\
\text { Deviation }\end{array}$ & $\begin{array}{l}\text { Skweness } \\
\text { coefficient }\end{array}$ \\
\hline \multirow{6}{*}{$\begin{array}{c}\text { Heart } \\
\text { and } \\
\text { blood } \\
\text { circle } \\
\text { functions }\end{array}$} & \multirow{3}{*}{$\begin{array}{l}\text { Rest } \\
\text { pulse }\end{array}$} & Experimental & 10 & 70.3 & 70 & 1.16 & 1.41 \\
\hline & & Control & 10 & $\mathbf{7 0}$ & 70 & 0.94 & $\mathbf{0}$ \\
\hline & & Overall & 20 & 70.15 & 70 & 1.04 & 0.92 \\
\hline & \multirow{3}{*}{$\begin{array}{c}\text { Pulse } \\
\text { reserve }\end{array}$} & Experimental & 10 & 129.6 & 130 & 1.71 & -1.53 \\
\hline & & Control & 10 & 130.1 & 130 & 0.74 & 1.91 \\
\hline & & Overall & 20 & 129.85 & 130 & 1.31 & -1.67 \\
\hline \multirow{6}{*}{$\begin{array}{c}\text { PWC } \\
170\end{array}$} & \multirow{3}{*}{ Absolute } & Experimental & 10 & 50.1 & 49.5 & 3.51 & 0.26 \\
\hline & & Control & 10 & 49.8 & 49.5 & 1.93 & 0.24 \\
\hline & & Overall & 20 & 49.95 & 49.5 & 2.76 & 0.36 \\
\hline & \multirow{3}{*}{ Relative } & Experimental & 10 & 0.65 & 0.65 & 0.04 & 0.09 \\
\hline & & Control & 10 & 0.64 & 0.64 & 0.06 & -0.34 \\
\hline & & Overall & 20 & 0.64 & 0.65 & 0.05 & -0.37 \\
\hline \multirow{3}{*}{\multicolumn{2}{|c|}{$\mathrm{VO}_{2} \max ($ Queen test) }} & Experimental & 10 & 158.7 & 156.5 & 22.41 & 0.21 \\
\hline & & Control & 10 & 150.6 & 154 & 15.58 & -0.17 \\
\hline & & Overall & 20 & 154.65 & 155.5 & 19.24 & 0.33 \\
\hline
\end{tabular}

Table (5) results show that skewness coefficient range from ( -1.67 to 2.02 ), indicating that measurements obtained are close to normal distribution where skewness coefficient values should be between \pm 3 and very close to zero This

confirms homogeneity of experimental, control, and overall research sample in physiological measurements before the experiment

Table (6)

statistical characterization for experimental, control and overall research sample in Scuba unit put on/off time (sec) before the experiment

\begin{tabular}{|c|c|c|c|c|c|c|}
\hline \multirow{2}{*}{ Tests } & \multirow[b]{2}{*}{ Groups } & \multirow[b]{2}{*}{$\mathbf{N}$} & \multicolumn{4}{|c|}{ Statistics significance } \\
\hline & & & Mean & Median & $\begin{array}{c}\text { Slandered } \\
\text { Deviation }\end{array}$ & $\begin{array}{l}\text { Skweness } \\
\text { coefficient }\end{array}$ \\
\hline \multirow{3}{*}{$\begin{array}{c}\text { Scuba unit put on/off } \\
\text { time (sec) }\end{array}$} & Experimental & 10 & 56.56 & $\mathbf{5 7 . 7 2}$ & 3.56 & -0.46 \\
\hline & Control & 10 & 55.27 & 54.29 & 3.59 & 0.37 \\
\hline & Overall & 20 & 55.91 & 55.75 & 3.54 & -0.04 \\
\hline
\end{tabular}

Table (6) results show that skewness coefficient range from ( -0.46 to 0.37$)$, indicating that measurements obtained are close to normal distribution where skewness coefficient values should be between \pm 3 and very close to zero This

confirms homogeneity of experimental, control, and overall research sample in Scuba unit put on/off time (sec) before the experiment.

Table ( 7)

Differences between experimental and control groups in physical tests before experiment ( homogeneity)

\begin{tabular}{|c|c|c|c|c|c|c|c|}
\hline \multirow[t]{2}{*}{ Statistics } & \multirow[b]{2}{*}{ Tests } & \multicolumn{2}{|c|}{$\begin{array}{l}\text { Experimental } \\
\text { group }(\mathrm{N}=10)\end{array}$} & \multicolumn{2}{|c|}{$\begin{array}{c}\text { Control } \\
\text { group }(\mathrm{N}=10)\end{array}$} & \multirow{2}{*}{$\begin{array}{c}\text { Differences } \\
\text { Between } \\
\text { averages }\end{array}$} & \multirow{2}{*}{ T Value } \\
\hline & & Mean & $\begin{array}{l}\text { Standard } \\
\text { Deviation }\end{array}$ & Mean & $\begin{array}{l}\text { Standard } \\
\text { Deviation }\end{array}$ & & \\
\hline \multirow{5}{*}{$\begin{array}{c}\text { Muscle } \\
\text { Strength } \\
\text { endurance }\end{array}$} & $400 \mathrm{~m}$ swimming time & 8.21 & 0.56 & 8.34 & 0.56 & 0.13 & 0.51 \\
\hline & Knee bent & 48 & 7.26 & 49.9 & 7.02 & 1.9 & 0.6 \\
\hline & Setting from lying & 36.8 & 5.53 & 35.4 & 4.74 & 1.4 & 0.61 \\
\hline & Trunk lifting & 33.6 & 7.18 & 33.9 & 7.99 & 0.3 & 0.09 \\
\hline & Arms bend & 21.9 & 3.11 & 20.4 & 3.34 & 1.5 & 1.04 \\
\hline \multirow{4}{*}{$\begin{array}{l}\text { Motion } \\
\text { Range }\end{array}$} & Foot adduction & 29.6 & 6.75 & 27.2 & 5.81 & 2.4 & 0.85 \\
\hline & Foot Extending & 26.6 & 7.6 & 25.5 & 4.97 & 1.1 & 0.38 \\
\hline & Hip adduction & 42.7 & 6.7 & 40 & 8.55 & 2.7 & 0.79 \\
\hline & Hip extending & 86.2 & 8.39 & 82.8 & 8.05 & 3.4 & 0.92 \\
\hline \multirow{2}{*}{ Speed } & Pendulum Running & 10.75 & 0.53 & 10.53 & 0.47 & 0.22 & 0.97 \\
\hline & Reaction speed & 0.23 & 0.02 & 0.23 & 0.01 & $\mathbf{0}$ & 0.22 \\
\hline Power & Vertical Jump & 32.9 & 3.38 & 33.2 & 3.39 & 0.3 & 0.2 \\
\hline Ability & Agility under water & 11.8 & 1.29 & 12.14 & 1.63 & 0.35 & 0.52 \\
\hline
\end{tabular}

* $\mathbf{T}$ significant at $\mathbf{0 . 0 5}$ level $=\mathbf{2 . 1 0}$ 
Table (7) results reveal that $\mathrm{T}$ value for differences between experimental and control groups ranged from ( 0.09 to 1.04 ) and these values are not significant at 0.05 level, which indicates homogeneity between the two groups in physical tests before experiment.

Table (8)

Differences between experimental and control groups in physiological measurements before experiment ( homogeneity)

\begin{tabular}{|c|c|c|c|c|c|c|c|}
\hline \multirow[t]{2}{*}{ Statistics } & \multirow[b]{2}{*}{ Tests } & \multicolumn{2}{|c|}{$\begin{array}{l}\text { Experimental } \\
\text { group }(\mathrm{N}=10)\end{array}$} & \multicolumn{2}{|c|}{$\begin{array}{c}\text { Control } \\
\text { group }(\mathrm{N}=10)\end{array}$} & \multirow{2}{*}{$\begin{array}{l}\text { Differences } \\
\text { Between } \\
\text { averages }\end{array}$} & \multirow{2}{*}{ T Value } \\
\hline & & Mean & $\begin{array}{l}\text { Standard } \\
\text { Deviation } \\
\end{array}$ & Mean & $\begin{array}{l}\text { Standard } \\
\text { Deviation } \\
\end{array}$ & & \\
\hline \multirow{7}{*}{$\begin{array}{c}\text { Lung } \\
\text { Function } \\
\text { Tests }\end{array}$} & V C & 7.09 & 0.65 & 6.97 & 1.2 & 0.13 & 0.29 \\
\hline & F V C & 7.03 & 0.61 & 6.75 & 1.12 & 0.28 & 0.71 \\
\hline & F E V 1 & 5.93 & 0.52 & 5.75 & 1 & 0.18 & 0.49 \\
\hline & FEV $1 \%$ & 84.48 & 5.94 & 85.51 & 9.64 & 1.03 & 0.29 \\
\hline & P E F & 12.11 & 1.27 & 12.27 & 1.17 & 0.16 & 0.29 \\
\hline & F I V C & 6.83 & 0.71 & 6.5 & 0.61 & 0.33 & 1.12 \\
\hline & M V V & 182 & 32.91 & 169.53 & 32.46 & 12.47 & 0.85 \\
\hline \multirow{2}{*}{$\begin{array}{l}\text { Heart and Blood circle } \\
\text { functions }\end{array}$} & Rest pulse & 70.3 & 1.16 & 70 & 0.94 & 0.3 & 0.63 \\
\hline & Pulse reserve & 129.6 & 1.71 & 130.1 & 0.74 & 0.5 & 0.85 \\
\hline \multirow{2}{*}{ PWC } & Absolute & 50.1 & 3.51 & 49.8 & 1.93 & 0.3 & 0.24 \\
\hline & Relative & 0.65 & 0.04 & 0.64 & 0.06 & 0.01 & 0.45 \\
\hline \multicolumn{2}{|c|}{$\mathrm{VO}_{2} \max ($ Queen test) } & 158.7 & 22.41 & 150.6 & 15.58 & 8.1 & 0.94 \\
\hline
\end{tabular}

$*$ T significant at 0.05 level $=2.10$

Table (8) results reveal that $\mathrm{T}$ value for differences between experimental and control groups ranged from (024 to 1.12) and these values are not significant at 0.05 level, which

indicates homogeneity between the two groups in physiological measurements before experiment.

Table (9)

Differences between experimental and control groups in Scuba unit put on/off time (sec) before experiment (homogeneity)

\begin{tabular}{|c|c|c|c|c|c|c|}
\hline \multirow[t]{2}{*}{ Statistics } & \multicolumn{2}{|c|}{$\begin{array}{l}\text { Experimental } \\
\text { group }(\mathrm{N}=10)\end{array}$} & \multicolumn{2}{|c|}{$\begin{array}{c}\text { Control } \\
\text { group }(\mathrm{N}=10)\end{array}$} & \multirow{2}{*}{$\begin{array}{c}\text { Differences } \\
\text { Between } \\
\text { averages }\end{array}$} & \multirow{2}{*}{ T Value } \\
\hline & Mean & $\begin{array}{l}\text { Standard } \\
\text { Deviation }\end{array}$ & Mean & $\begin{array}{l}\text { Standard } \\
\text { Deviation }\end{array}$ & & \\
\hline Performance time & 56.56 & 3.56 & 55.27 & 3.59 & 1.29 & 0.81 \\
\hline
\end{tabular}

Table (9) results reveal that $\mathrm{T}$ value for differences between experimental and control groups was 0.81 and this values is not significant at 0.05 level, which indicates homogeneity between the two groups in physiological measurements before experiment.

Table (10)

Differences between pre and post measurements for experimental group in physical tests $(n=10)$

\begin{tabular}{|c|c|c|c|c|c|c|c|c|c|}
\hline \multirow[t]{2}{*}{ Statistics } & \multirow[b]{2}{*}{ Tests } & \multicolumn{2}{|c|}{ Pre measurement } & \multicolumn{2}{|c|}{$\begin{array}{c}\text { Post } \\
\text { measurement }\end{array}$} & \multicolumn{2}{|c|}{$\begin{array}{c}\text { Differences } \\
\text { Between } \\
\text { averages } \\
\end{array}$} & \multirow{2}{*}{$\begin{array}{c}\text { T } \\
\text { Value }\end{array}$} & \multirow{2}{*}{$\begin{array}{c}\text { Improvement } \\
\text { Percentage } \\
\%\end{array}$} \\
\hline & & స్ట & 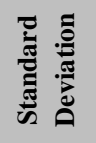 & 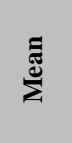 & 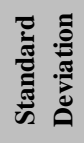 & $\stackrel{\Xi}{\Sigma}$ & 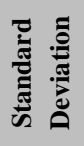 & & \\
\hline \multirow{5}{*}{$\begin{array}{c}\text { Muscle } \\
\text { Strength } \\
\text { endurance }\end{array}$} & $400 \mathrm{~m}$ swimming time & 8.21 & 0.56 & 7.15 & 0.5 & 1.06 & 0.4 & $8.36^{*}$ & 12.86 \\
\hline & Knee bent & 48 & 7.26 & 63.1 & 4.41 & 15.1 & 8.41 & $5.68 *$ & 31.46 \\
\hline & Setting from lying & 36.8 & 5.53 & 45.5 & 3.06 & 8.7 & 5.72 & $4.81 *$ & 23.64 \\
\hline & Trunk lifting & 33.6 & 7.18 & 43.4 & 4.35 & 9.8 & 7.41 & $4.18^{*}$ & 29.17 \\
\hline & Arms bend & 21.9 & 3.11 & 31.9 & 2.47 & 10 & 4.29 & $7.36 *$ & 45.66 \\
\hline Motion & Foot adduction & 29.6 & 6.75 & 48 & 7.89 & 18.4 & 5.44 & $10.69 *$ & 62.16 \\
\hline
\end{tabular}




\begin{tabular}{|c|c|c|c|c|c|c|c|c|c|}
\hline \multirow[t]{2}{*}{ Statistics } & \multirow[b]{2}{*}{ Tests } & \multicolumn{2}{|c|}{ Pre measurement } & \multicolumn{2}{|c|}{$\begin{array}{c}\text { Post } \\
\text { measurement }\end{array}$} & \multicolumn{2}{|c|}{$\begin{array}{c}\text { Differences } \\
\text { Between } \\
\text { averages }\end{array}$} & \multirow{2}{*}{$\begin{array}{c}\text { T } \\
\text { Value }\end{array}$} & \multirow{2}{*}{$\begin{array}{c}\text { Improvemen } \\
\text { Percentage } \\
\%\end{array}$} \\
\hline & & $\sum_{\Sigma}^{\Xi}$ & 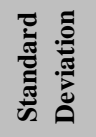 & $\sum_{\Sigma}^{\Xi}$ & 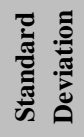 & $\stackrel{\Xi}{\bar{E}}$ & 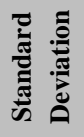 & & \\
\hline \multirow[t]{3}{*}{ Range } & Foot Extending & 26.6 & 7.6 & 44.4 & 10.45 & 17.8 & 10.66 & $5.28 *$ & 66.92 \\
\hline & Hip adduction & 42.7 & 6.7 & 52 & 4.62 & 9.3 & 4.55 & $6.47 *$ & 21.78 \\
\hline & Hip extending & 86.2 & 8.39 & 95.6 & 12.93 & 9.4 & 6.4 & $4.65 *$ & 10.9 \\
\hline \multirow{2}{*}{ Speed } & Pendulum Running & 10.75 & 0.53 & 9.73 & 0.43 & 1.02 & 0.63 & $5.16 *$ & 9.51 \\
\hline & Reaction speed & 0.23 & 0.02 & 0.21 & 0.01 & 0.03 & 0.03 & $3.16^{*}$ & 10.98 \\
\hline Power & Vertical Jump & 32.9 & 3.38 & 51 & 9.25 & 18.1 & 10.05 & $5.70 *$ & 55.02 \\
\hline Ability & Agility under water & 11.8 & 1.29 & 9.4 & 0.88 & 2.4 & 0.79 & $9.63^{*}$ & 20.37 \\
\hline
\end{tabular}

* $\mathbf{T}$ significant at $\mathbf{0 . 0 5}$ level $=\mathbf{2 . 2 6}$

Table (10) results reveal that $\mathrm{T}$ test value for differences between pre and post measurements ranged between ( 3.16 to 10.69 ), and these values are greater than the T significant

value at 0.05 level, and improvement percentage ranged between $(9.51 \%-66.92 \%)$

Table (11)

Differences between pre and post measurements for experimental group in physiological measurements

$(\mathbf{n}=10)$

\begin{tabular}{|c|c|c|c|c|c|c|c|c|c|}
\hline \multirow[t]{2}{*}{ Statistics } & \multirow[b]{2}{*}{ Tests } & \multicolumn{2}{|c|}{ Pre measurement } & \multicolumn{2}{|c|}{ Post measurement } & \multicolumn{2}{|c|}{$\begin{array}{c}\text { Differences } \\
\text { Between } \\
\text { averages } \\
\end{array}$} & \multirow[t]{2}{*}{ T Value } & \multirow{2}{*}{$\begin{array}{c}\text { Improvement } \\
\text { Percentage } \\
\%\end{array}$} \\
\hline & & Mean & $\begin{array}{l}\text { Standard } \\
\text { Deviation } \\
\end{array}$ & Mean & $\begin{array}{l}\text { Standard } \\
\text { Deviation } \\
\end{array}$ & Mean & $\begin{array}{l}\text { Standard } \\
\text { Deviation } \\
\end{array}$ & & \\
\hline \multirow{7}{*}{$\begin{array}{c}\text { Lung } \\
\text { Function } \\
\text { Tests }\end{array}$} & V C & 7.09 & 0.65 & 7.73 & 0.79 & 0.63 & 0.46 & 4.41* & 8.94 \\
\hline & F V C & 7.03 & 0.61 & 8.91 & 0.76 & 1.88 & 1.12 & $5.31 *$ & 26.67 \\
\hline & F E V 1 & 5.93 & 0.52 & 7.19 & 0.54 & 1.26 & 0.72 & 5.53* & 21.33 \\
\hline & FEV $1 \%$ & 84.48 & 5.94 & 87.84 & 5.5 & 3.36 & 6.45 & 1.65 & 3.98 \\
\hline & P E F & 12.11 & 1.27 & 13.34 & 0.64 & 1.23 & 1.63 & $2.40 *$ & 10.16 \\
\hline & F I V C & 6.83 & 0.71 & 7.98 & 0.52 & 1.15 & 0.98 & $3.70^{*}$ & 16.8 \\
\hline & M V V & 182 & 32.91 & 231.95 & 32.3 & 49.95 & 23.45 & $6.73 *$ & 27.45 \\
\hline \multirow{2}{*}{$\begin{array}{c}\text { Heart and Blood } \\
\text { circle } \\
\text { Functions }\end{array}$} & Rest pulse & 70.3 & 1.16 & 66.8 & 0.42 & 3.5 & 1.18 & 9.39* & 4.98 \\
\hline & Pulse reserve & 129.6 & 1.71 & 137.93 & 5.62 & 8.33 & 6.3 & $4.18^{*}$ & 6.43 \\
\hline \multirow{2}{*}{ PWC } & Absolute & 50.1 & 3.51 & 61.72 & 4.42 & 11.62 & 3.87 & $9.49 *$ & 23.19 \\
\hline & Relative & 0.65 & 0.04 & 0.8 & 0.07 & 0.15 & 0.05 & $8.83 *$ & 23.35 \\
\hline \multicolumn{2}{|c|}{$\mathrm{VO}_{2} \max ($ Queen test) } & 158.7 & 22.41 & 241.6 & 38.53 & 82.9 & 41.64 & $6.30^{*}$ & 52.24 \\
\hline
\end{tabular}

* $\mathbf{T}$ significant at 0.05 level $=\mathbf{2 . 2 6}$

Table (11) results reveal that $\mathrm{T}$ test value for differences between pre and post measurements ranged between ( 2.40 to 9.49), and these values are greater than the $\mathrm{T}$ significant value at 0.05 level, and improvement percentage ranged between $(3.98 \%-52.24 \%)$ 
Table (12)

Differences between pre and post measurements for experimental group in Scuba unit put on/off time

$(\mathbf{n}=10)$

\begin{tabular}{|r|c|c|c|c|c|c|c|c|}
\hline \multirow{2}{*}{ Statistics } & \multicolumn{2}{|c|}{ Pre measurement } & \multicolumn{2}{|c|}{ Post measurement } & \multicolumn{2}{|c|}{$\begin{array}{c}\text { Differences } \\
\text { Between } \\
\text { averages }\end{array}$} & \multirow{2}{*}{ T Value } & $\begin{array}{c}\text { Improvement } \\
\text { Percentage } \\
\%\end{array}$ \\
\cline { 2 - 8 } & Mean & $\begin{array}{c}\text { Standard } \\
\text { Deviation }\end{array}$ & Mean & $\begin{array}{c}\text { Standard } \\
\text { Deviation }\end{array}$ & Mean & $\begin{array}{c}\text { Standard } \\
\text { Deviation }\end{array}$ & & \\
\hline Performance time & 56.56 & 3.56 & 40.63 & 5.78 & 15.93 & 5.2 & 9.69 & 28.17 \\
\hline
\end{tabular}

Table (12) results reveal that $\mathrm{T}$ test value for differences between pre and post measurements was (9.69), and this value are greater than the $\mathrm{T}$ significant value at 0.05 level, and improvement percentage was $(28.17 \%)$

Table (13)

Differences between pre and post measurements for control group in physical tests $(n=10)$

\begin{tabular}{|c|c|c|c|c|c|c|c|c|c|}
\hline \multirow[t]{2}{*}{ Statistics } & \multirow[b]{2}{*}{ Tests } & \multicolumn{2}{|c|}{ Pre measurement } & \multicolumn{2}{|c|}{ Post measurement } & \multicolumn{2}{|c|}{$\begin{array}{c}\text { Differences } \\
\text { Between } \\
\text { averages }\end{array}$} & \multirow{2}{*}{$\begin{array}{c}\text { T } \\
\text { Value }\end{array}$} & \multirow{2}{*}{$\begin{array}{c}\text { Improvement } \\
\text { Percentage } \\
\%\end{array}$} \\
\hline & & $\stackrel{\Xi}{\Sigma}$ & $\begin{array}{l}\text { Standard } \\
\text { Deviation }\end{array}$ & $\stackrel{\Xi}{\Xi}$ & $\begin{array}{l}\text { Standard } \\
\text { Deviation }\end{array}$ & $\sum^{\Xi}$ & $\begin{array}{l}\text { Standard } \\
\text { Deviation }\end{array}$ & & \\
\hline \multirow{5}{*}{ 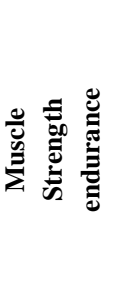 } & $400 \mathrm{~m}$ swimming time & 8.34 & 0.56 & 8.28 & 0.54 & 0.06 & 0.17 & 1.04 & 0.66 \\
\hline & Knee bent & 49.9 & 7.02 & 54.5 & 7.63 & 4.6 & 3.24 & $4.49 *$ & 9.22 \\
\hline & Setting from lying & 35.4 & 4.74 & 38.7 & 5.44 & 3.3 & 1.83 & $5.71 *$ & 9.32 \\
\hline & Trunk lifting & 33.9 & 7.99 & 36.5 & 8.32 & 2.6 & 2.67 & $3.07 *$ & 7.67 \\
\hline & Arms bend & 20.4 & 3.34 & 22 & 2.67 & 1.6 & 1.78 & $2.85 *$ & 7.84 \\
\hline \multirow{4}{*}{$\begin{array}{c}\text { Motion } \\
\text { Range }\end{array}$} & Foot adduction & 27.2 & 5.81 & 31.3 & 4.19 & 4.1 & 3.84 & $3.37 *$ & 15.07 \\
\hline & Foot Extending & 25.5 & 4.97 & 30 & 5.77 & 4.5 & 4.38 & $3.25 *$ & 17.65 \\
\hline & Hip adduction & 40 & 8.55 & 41.4 & 7.78 & 1.4 & 4.74 & 0.93 & 3.5 \\
\hline & Hip extending & 82.8 & 8.05 & 85.4 & 8.45 & 2.6 & 1.65 & $4.99 *$ & 3.14 \\
\hline \multirow{2}{*}{ Speed } & Pendulum Running & 10.53 & 0.47 & 10.44 & 0.42 & 0.09 & 0.38 & 0.72 & 0.82 \\
\hline & Reaction speed & 0.23 & 0.01 & 0.23 & 0.01 & $\mathbf{0}$ & $\mathbf{0}$ & $\mathbf{0}$ & 0 \\
\hline Power & Vertical Jump & 33.2 & 3.39 & 34.4 & 3.63 & 1.2 & 3.97 & 0.96 & 3.61 \\
\hline Ability & Agility under water & 12.14 & 1.63 & 11.54 & 1.24 & 0.6 & 0.68 & $2.80 *$ & 4.93 \\
\hline
\end{tabular}

* $\mathbf{T}$ significant at $\mathbf{0 . 0 5}$ level $=\mathbf{2 . 2 6}$

Table (13) results reveal that $\mathrm{T}$ test value for differences between pre and post measurements for most measurements ranged between $(2.80-5.71)$, and these values are greater than the $\mathrm{T}$ significant value at 0.05 level, there was no differences between pre and post measurements in $400 \mathrm{~m}$ swimming time, Hip adduction, Pendulum Running, Reaction speed and Vertical Jump, improvement percentage ranged between ( $0.00 \%-17.65 \%$ ) 
Table (14)

Differences between pre and post measurements for control group in physiological measurements (n=10)

\begin{tabular}{|c|c|c|c|c|c|c|c|c|c|}
\hline \multirow[t]{2}{*}{ Statistics } & \multirow[b]{2}{*}{ Tests } & \multicolumn{2}{|c|}{ Pre measurement } & \multicolumn{2}{|c|}{ Post measurement } & \multicolumn{2}{|c|}{$\begin{array}{c}\text { Differences } \\
\text { Between } \\
\text { averages } \\
\end{array}$} & \multirow{2}{*}{$\begin{array}{c}\text { T } \\
\text { Value }\end{array}$} & \multirow{2}{*}{$\begin{array}{c}\text { Improvement } \\
\text { Percentage } \\
\%\end{array}$} \\
\hline & & $\sum^{\mathbb{E}}$ & $\begin{array}{l}\text { Standard } \\
\text { Deviation }\end{array}$ & $\sum_{\Sigma}^{\Xi \Xi}$ & $\begin{array}{l}\text { Standard } \\
\text { Deviation }\end{array}$ & $\stackrel{\Xi}{\mathbb{E}}$ & $\begin{array}{l}\text { Standard } \\
\text { Deviation }\end{array}$ & & \\
\hline \multirow{7}{*}{$\begin{array}{c}\text { Lung } \\
\text { Function } \\
\text { Tests }\end{array}$} & V C & 6.97 & 1.2 & 7.19 & 0.76 & 0.22 & 1.07 & 0.65 & 3.14 \\
\hline & F V C & 6.75 & 1.12 & 7.21 & 1.02 & 0.46 & 0.61 & $2.36 *$ & 6.79 \\
\hline & F E V 1 & 5.75 & 1 & 5.95 & 0.59 & 0.19 & 0.94 & 0.65 & 3.39 \\
\hline & FEV $1 \%$ & 85.51 & 9.64 & 82.37 & 6.38 & 3.14 & 9.56 & 1.04 & 3.67 \\
\hline & P E F & 12.27 & 1.17 & 10.14 & 1.81 & 2.13 & 1.71 & $3.93 *$ & 17.35 \\
\hline & F I V C & 6.5 & 0.61 & 7.08 & 0.64 & 0.58 & 0.66 & $2.78 *$ & 8.92 \\
\hline & M V V & 169.53 & 32.46 & 189.2 & 33.32 & 19.67 & 39.05 & 1.59 & 11.6 \\
\hline \multirow{2}{*}{$\begin{array}{c}\text { Heart and } \\
\text { Blood circle } \\
\text { Functions }\end{array}$} & Rest pulse & 70 & 0.94 & 69.4 & 0.97 & 0.6 & 0.7 & $2.71 *$ & 0.86 \\
\hline & Pulse reserve & 130.1 & 0.74 & 131.33 & 0.62 & 1.23 & 0.88 & $4.38 *$ & 0.94 \\
\hline \multirow{2}{*}{ PWC } & Absolute & 49.8 & 1.93 & 48.84 & 3.2 & 0.96 & 3.39 & 0.9 & 1.93 \\
\hline & Relative & 0.64 & 0.06 & 0.62 & 0.07 & 0.01 & 0.04 & 0.87 & 1.94 \\
\hline \multicolumn{2}{|c|}{$\mathrm{VO}_{2} \max$ (Queen test) } & 150.6 & 15.58 & 204.9 & 37.31 & 54.3 & 26.64 & $6.45^{*}$ & 36.06 \\
\hline
\end{tabular}

* $\mathbf{T}$ significant at 0.05 level $=\mathbf{2 . 2 6}$

Table (15) results reveal that $\mathrm{T}$ test value for differences between pre and post measurements were significant in 6 tests only and ranged between ( $2.36-6.45$ ), and these values are greater than the $\mathrm{T}$ significant value at 0.05 level, while differences were not significant in 6 other test where $\mathrm{T}$ value ranged between $(0.065-1.59)$, and these values are lower than the $\mathrm{T}$ significant value at 0.05 level and improvement percentage ranged between $(0.94 \%$ to $36.06 \%)$

Table (15)

Differences between pre and post measurements for control group in Scuba unit put on/off time (n=10)

\begin{tabular}{|c|c|c|c|c|c|c|c|c|}
\hline \multirow[t]{2}{*}{ Statistics } & \multicolumn{2}{|c|}{ Pre measurement } & \multicolumn{2}{|c|}{ Post measurement } & \multicolumn{2}{|c|}{$\begin{array}{c}\text { Differences } \\
\text { Between } \\
\text { averages }\end{array}$} & \multirow{2}{*}{ T Value } & \multirow{2}{*}{$\begin{array}{c}\text { Improvement } \\
\text { Percentage } \\
\%\end{array}$} \\
\hline & Mean & $\begin{array}{l}\text { Standard } \\
\text { Deviation }\end{array}$ & Mean & $\begin{array}{l}\text { Standard } \\
\text { Deviation }\end{array}$ & Mean & $\begin{array}{l}\text { Standard } \\
\text { Deviation }\end{array}$ & & \\
\hline Performance time & 55.27 & 3.59 & 50.05 & 3.73 & 5.22 & 1.13 & $14.57 *$ & 9.44 \\
\hline
\end{tabular}

* $\mathbf{T}$ significant at 0.05 level $=\mathbf{2 . 2 6}$

Table (15) results reveal that $\mathrm{T}$ test value for differences between pre and post measurements was (9.44), and this value are greater than the $\mathrm{T}$ significant value at 0.05 level, and improvement percentage was $(9.44 \%)$ 
Table (16)

Differences between experimental and control groups in physical tests after experiment

\begin{tabular}{|c|c|c|c|c|c|c|c|c|}
\hline \multirow[t]{2}{*}{ Statistics } & \multirow[b]{2}{*}{ Tests } & \multicolumn{2}{|c|}{$\begin{array}{l}\text { Experimental } \\
\text { group }(\mathrm{N}=10)\end{array}$} & \multicolumn{2}{|c|}{$\begin{array}{c}\text { Control } \\
\text { group }(\mathrm{N}=10)\end{array}$} & \multirow{2}{*}{$\begin{array}{c}\text { Differences } \\
\text { Between } \\
\text { Averages }\end{array}$} & \multirow{2}{*}{$\begin{array}{c}\text { T } \\
\text { Value }\end{array}$} & \multirow{2}{*}{$\begin{array}{c}\text { Differences } \\
\text { Percentage } \\
\%\end{array}$} \\
\hline & & Mean & $\begin{array}{l}\text { Standard } \\
\text { Deviation }\end{array}$ & Mean & $\begin{array}{l}\text { Standard } \\
\text { Deviation }\end{array}$ & & & \\
\hline \multirow{5}{*}{$\begin{array}{c}\text { Muscle } \\
\text { Strength } \\
\text { endurance }\end{array}$} & $400 \mathrm{~m}$ swimming time & 7.15 & 0.5 & 8.28 & 0.54 & 1.13 & $4.83 *$ & 15.78 \\
\hline & Knee bent & 63.1 & 4.41 & 54.5 & 7.63 & 8.6 & $3.09 *$ & 13.63 \\
\hline & Setting from lying & 45.5 & 3.06 & 38.7 & 5.44 & 6.8 & $3.45 *$ & 14.95 \\
\hline & Trunk lifting & 43.4 & 4.35 & 36.5 & 8.32 & 6.9 & $2.32 *$ & 15.9 \\
\hline & Arms bend & 31.9 & 2.47 & 22 & 2.67 & 9.9 & $8.61 *$ & 31.03 \\
\hline \multirow{4}{*}{$\begin{array}{l}\text { Motion } \\
\text { Range }\end{array}$} & Foot adduction & 48 & 7.89 & 31.3 & 4.19 & 16.7 & $5.91 *$ & 34.79 \\
\hline & Foot Extending & 44.4 & 10.45 & 30 & 5.77 & 14.4 & $3.81 *$ & 32.43 \\
\hline & Hip adduction & 52 & 4.62 & 41.4 & 7.78 & 10.6 & $3.71 *$ & 20.38 \\
\hline & Hip extending & 95.6 & 12.93 & 85.4 & 8.45 & 10.2 & $2.11 *$ & 10.67 \\
\hline \multirow{2}{*}{ Speed } & Pendulum Running & 9.73 & 0.43 & 10.44 & 0.42 & 0.72 & $3.79 *$ & 7.39 \\
\hline & Reaction speed & 0.21 & 0.01 & 0.23 & 0.01 & 0.02 & $5.21 *$ & 11.56 \\
\hline Power & Vertical Jump & 51 & 9.25 & 34.4 & 3.63 & 16.6 & $5.28 *$ & 32.55 \\
\hline Ability & Agility under water & 9.4 & 0.88 & 11.54 & 1.24 & 2.15 & $4.49 *$ & 22.88 \\
\hline
\end{tabular}

* $\mathbf{T}$ significant at 0.05 level $=\mathbf{2 . 1 0}$

Table (16) results reveal existent of significant differences between experimental and control groups after experiments in all tests as that $\mathrm{T}$ value ranged between $(2.11-8.61)$ and these values were significant at 0.05 level, differences percentage ranged between $(7.39 \%-34.79 \%)$.

Table (17)

Differences between experimental and control groups in physiological measurements after experiment

\begin{tabular}{|c|c|c|c|c|c|c|c|c|}
\hline \multirow[t]{2}{*}{ Statistics } & \multirow[b]{2}{*}{ Tests } & \multicolumn{2}{|c|}{$\begin{array}{l}\text { Experimental } \\
\text { group }(N=10)\end{array}$} & \multicolumn{2}{|c|}{$\begin{array}{c}\text { Control } \\
\text { group }(N=10)\end{array}$} & \multirow{2}{*}{$\begin{array}{c}\text { Differences } \\
\text { Between } \\
\text { averages }\end{array}$} & \multirow{2}{*}{ T Value } & \multirow{2}{*}{$\begin{array}{c}\text { Differences } \\
\text { Percentage } \\
\%\end{array}$} \\
\hline & & Mean & $\begin{array}{l}\text { Standard } \\
\text { Deviation }\end{array}$ & Mean & $\begin{array}{l}\text { Standard } \\
\text { Deviation }\end{array}$ & & & \\
\hline \multirow{7}{*}{$\begin{array}{c}\text { Lung } \\
\text { Function } \\
\text { Tests }\end{array}$} & V C & 7.73 & 0.79 & 7.19 & 0.76 & 0.54 & 1.56 & 6.99 \\
\hline & F V C & 8.91 & 0.76 & 7.21 & 1.02 & 1.7 & $4.22 *$ & 19.1 \\
\hline & F E V 1 & 7.19 & 0.54 & 5.95 & 0.59 & 1.25 & $4.91 *$ & 17.31 \\
\hline & FEV $1 \%$ & 87.84 & 5.5 & 82.37 & 6.38 & 5.47 & 2.06 & 6.23 \\
\hline & P E F & 13.34 & 0.64 & 10.14 & 1.81 & 3.2 & $5.29 *$ & 24.01 \\
\hline & F I V C & 7.98 & 0.52 & 7.08 & 0.64 & 0.9 & $3.45 *$ & 11.28 \\
\hline & M V V & 231.95 & 32.3 & 189.2 & 33.32 & 42.75 & $2.91 *$ & 18.43 \\
\hline \multirow{2}{*}{$\begin{array}{l}\text { Heart and Blood circle } \\
\text { functions }\end{array}$} & Rest pulse & 66.8 & 0.42 & 69.4 & 0.97 & 2.6 & $7.80 *$ & 3.89 \\
\hline & Pulse reserve & 137.93 & 5.62 & 131.33 & 0.62 & 6.6 & $3.69 *$ & 4.79 \\
\hline \multirow{2}{*}{ PWC } & Absolute & 61.72 & 4.42 & 48.84 & 3.2 & 12.88 & $7.46^{*}$ & 20.87 \\
\hline & Relative & 0.8 & 0.07 & 0.62 & 0.07 & 0.17 & $5.65 *$ & 21.81 \\
\hline \multicolumn{2}{|c|}{$\mathrm{VO}_{2} \max ($ Queen test) } & 241.6 & 38.53 & 204.9 & 37.31 & 36.7 & $2.16 *$ & 15.19 \\
\hline
\end{tabular}

* $\mathbf{T}$ significant at $\mathbf{0 . 0 5}$ level $=\mathbf{2 . 1 0}$

Table (17) results reveal existent of significant differences between experimental and control groups after experiments in all physiological measurements (except two measurements) tests as that $\mathrm{T}$ value ranged between (2.167.80) and these values are significant at 0.05 level, differences percentage ranged between $(3.89 \%-24.01 \%)$. 
Table (18)

Differences between experimental and control groups in Scuba unit put on/off time (sec) after experiment

\begin{tabular}{|c|c|c|c|c|c|c|}
\hline \multirow{2}{*}{$\begin{array}{c}\text { Statistics } \\
\text { Tests }\end{array}$} & \multicolumn{2}{|c|}{$\begin{array}{l}\text { Experimental } \\
\text { group }(\mathbf{N}=10)\end{array}$} & \multicolumn{2}{|c|}{$\begin{array}{c}\text { Control } \\
\text { group }(N=10)\end{array}$} & \multirow{2}{*}{$\begin{array}{c}\text { Differences } \\
\text { Between } \\
\text { averages }\end{array}$} & \multirow{2}{*}{ T Value } \\
\hline & Mean & $\begin{array}{l}\text { Standard } \\
\text { Deviation }\end{array}$ & Mean & $\begin{array}{l}\text { Standard } \\
\text { Deviation }\end{array}$ & & \\
\hline Performance time & 40.63 & 5.78 & 50.05 & 3.73 & 9.42 & $4.33 *$ \\
\hline
\end{tabular}

Table (18) results reveal existent of significant differences between experimental and control groups after experiments in Scuba unit put on/off time as that $\mathrm{T}$ value ranged was (4.3380) which is significant at 0.05 level, differences percentage ranged between $(3.89 \%-24.01 \%)$.

\section{Discussion:}

The extent physical responses depend on vital body system functional development, where research results indicate the positive impact of training on improving fitness level, Table (16) shows the significant superiority of experimental group on control group at 0.05 level in physical tests after experiment; which is attributed to the improved physical and functional status of vital body systems as a result of regularity in physical preparation programs and different intensity step exercise program accompanied with music for a period of (10) weeks and also diversity in exercise forms in the proposed program, which requires involvement of arms and legs in multiple directions , Brick (2001, 502), Westcott $(2002,167)$ Kostich study (2002) indicate that step exercises have positive effect on improving individual's physical fitness level, thereby first hypothesis could be accepted, where the experimental group outperformed the control group in post-physical measurements .

In light of the differences between experimental group control group in physiological measurements after the experiment, it is clear from table (17) that there are significant differences at the 0.05 level in all physiological measurements in favor of experimental group except for vital capacity (VC) and forced expiratory volume of air \% $\left(\mathrm{FEV}_{1} \%\right)$, where improvement percentage were $6.99 \%$, $6.23 \%$, respectively, Researcher attributes significant differences in some of respiratory functions in favor of experimental group to respiratory system efficiency and effectiveness positive development as one of the manifestations of adoption for proposed program, which directed to affect circulatory and respiratory system, these results are consistent with what referred by Eastwood et al (2001, 95) about effect of sports training to improve some physiological measurements, and what previously mentioned show superiority of experimental group over control the post measurement for physiological variables, and so second hypothesis could be accepted: There are statistically significant differences between pre and post measurements in some functional responses level for experimental and control group in favor of experimental group.

Comparing the differences between experimental group and control group in performance time (scuba unit put on / off) after the experiment show that progress in physical, and physiological tests positively affect skill performance time as shown in the table (18), where $\mathrm{T}$ value was 4.33 which is significant at 0.05 level in favor of experimental group, the study confirms Kostsh (2002), Mustafa Noor et al ( 2002), Wylagala et al (2007) studies confirm that use of step exercises lead to upgrading physical, and physiological level, which in turn affect skill performance level, and so third hypotheses could be accepted: There are statistically significant differences between pre and post measurements in improving performance time for experimental and control group in favor of experimental group.

\section{Conclusions:}

- The proposed training program for different intensity step exercises accompanied with music has positive effect on improving some physical abilities level, functional responses - divers' skill performance level.

\section{Recommendations:}

- It is possible to employ different intensity step exercises accompanied with music in physical efficiency development training with what it include from aerobic and anaerobic abilities by controlling performance rates and performed step exercises' nature.

- Using varied load (wave) training method) to improve physical and functional abilities, and performance level in some sports activities.

- Using step box as training mean because of its positive impact in improving physical and functional abilities, and skill performance level

\section{References}

1. Abeer Ramadan (2000) : The impact of development of respiratory endurance using step box on some physiological variables and 800 meter running time, unpublished Master Thesis 
,Faculty of Physical Education for Girls , Alexandria University . (in Arabic language)

2. Afaf Darwish, Nourhanne Hassan (1997) : Scientific and practical foundations of using step box in aerobic exercise, Dar EL Maaref, Alexandria. (in Arabic language)

3. Azmi Mr. Faisal (2002) : Step exercises effectiveness on developing aerobic and anaerobic energy systems for age stage 13-15 years, unpublished Master Thesis, Faculty of Physical Education for Men, Alexandria University . (in Arabic language)

4. Bastawissi Ahmed Bastawissi (1999) : sports training principles and theories, Dar ELFekr ElArabi, Cairo .(in Arabic LAnguage

5. Brick, L . (2001) : Fitness aerobic - fitness spectrum series, human kinetics publishers, inc Hong Kong.

6. Champion,N., Hurst,G., (2000) : The Aerobics Instructor's Handbook, A\&C Black, London.

7. Corbin, C.B. \& Lindesy, R. (2000) : Concepts of physical fitness with laboratories, atimes mirror higher education group, inc , U.S.A

8. Eastwood, P.R., Hillman ,D.R., Finucane , R.E. (2001) : Inspiratory muscle performance in endurance athletes and sedentary subjects, respirology J-, jun.vol.6 (2) .pp.95-104

9. Faisal. A . Keith Beavers, Andrew Robertson, Richard Hughson (2009) : Prior moderate and heavy exercise accelerate oxygen uptake and cardiac output kinetics in endurance athletes. $J$ Appl. Physiol. 106: 1553-1563.

10. Foss,M.L. ， Keteyian,S .J, (2002) : Fox's physiological basis for exercise and sport ,6thed.,Mc Graw -Hill co., Inc, Singapore.

11. Iman Abdullah (2000) : A study of some physiological, physical and psychological responses for aerobic exercise program using step box, unpublished Master Thesis , Faculty of Physical Education for Girls , Alexandria University .(in Arabic language)

12. Kostich ,A ., (2002): Used properly fins can be a boost to your Swim Strength of Technique Sep.

13. Levy. A.M. , Furst . M.L. , (2002): Sports injury Hand book, professional Advice for Amateur Athletes, John Wiley \& sons , Inc. , U.S.A

14. Magdy Mohamed Abu Zaid (2007) : Modern training programs - Swimming competitions disables diving, Faculty of Physical Education for Men, Alexandria University. (in Arabic Language)

15. Mahmoud Ibrahim Marei (2001) : Impact of exercise program using one of the learning technology means on some variables associated with compulsory exercise phrase for Faculty of Physical Education students, unpublished Master Thesis , Faculty of Physical Education for Men, Alexandria University . (in Arabic Language)

16. Mustafa Noor, Mohammad Azab, Ahmed AlMuhammadi (2002) : A study of some functional responses to different intensity step exercise within junior swimmers, scientific production , the scientific journal of Physical Education and Sports, Menoufia University . (in Arabic Language)

17. Sharkey,B . J., (2001): Fitness and Health 4th ed. , Human Kinetics , U.S.A.

18. Westcott, W. , (2002): Building Strength and Stamina, New Nautilus Training for total fitness , Nautilus international , Human Kinetics , Inc. , U.S.A.

19. Wylagala et-al (2007): The development of respiratory muscles during exercise and its effect on swimming endurance in divers, U.S.A 